\title{
Two-dimensional, regularised inversion of VLF data
}

\author{
David Beamish \\ British Geological Survey, Keyworth, Nottingham NG I 2 5GG, UK
}

Beamish, D., 1994. Two-dimensional, regularised inversion of VLF data. Journal of Applied Geophysics 32, 357-374.

DOI: 10.1016/0926-9851(94)90034-5

\begin{abstract}
Very low frequency electromagnetic (EM) methods using VLF transmitters have found many applications in subsurface geophysical investigations. Surface measurements involving both the vertical component of the magnetic field (VLF-EM or VLF-Z) and of the apparent resistivity (VLF-R) are increasingly common. Although extensive VLF data sets have been successfully used for mapping purposes, modelling and interpretation techniques which assess the third (i.e. depth) dimension appear limited. Given a profile of VLF-R measurements the main purpose of the present study is to demonstrate an automatic method for the construction of a resistivity cross-section. The technique used is one of a new generation of regularised inversion methods. These techniques attempt to overcome the problem of equivalence/non-uniqueness in EM sounding data by constructing the resistivity distribution with the minimum amount of structure that fits the data. VLF data represent a special case of plane-wave EM sounding in that they conform, in practice, to a single-frequency technique. This fact imposes a limitation in the amount of vertical resolution that we can expect using such data. In the case of two-dimensional modelling and inversion, resolution through the cross-section is a resultant attribute from both vertical and lateral resistivity gradients within the subsurface. In order to provide insight into the practical application of regularised inversion techniques to VLF data, both synthetic and field examples are considered. Both sets of examples are primarily concerned with VLF data applied to near-surface fault mapping where the main aim is to assess the location, dip and depth extent of conductive subsurface features.
\end{abstract}




\section{Introduction}

The VLF survey technique is a well-established electromagnetic (EM) method of applied geophysics. Its use for geological and hydrogeological applications has been reviewed by McNeill (1990). The method is one of a class of EM techniques that conform to a planewave sounding of subsurface resistivity structure; a closely allied (natural-field) technique is the audiomagnetotellurics (AMT) method (e.g. Strangway et al., 1973). The VLF-EM technique makes use of one or more distant radio transmitters operating between 15 and $30 \mathrm{kHz}$. The limited bandwidth means that, although several measurements may be obtained at different frequencies (using different transmitters), a main attribute of the method is that of a single frequency sounding. The lack of bandwidth is compensated for by the fact that the instrumentation is very portable and cost-effective. An extensive surveying capability is provided by a single, roving operator.

The VLF method was developed as an inductive sounding technique measuring the amplitude and (subsequently) phase relationship between the vertical (secondary) magnetic field ( $Z$ ) relative to the horizontal primary field $(\mathrm{H})$. This method, referred to here as VLF-Z, relies on wavefield interaction with two-dimensional (2-D) and three-dimensional (3-D) resistivity structure. The technique has since been extended to include a measure of the induced horizontal electric field (E) component. This VLF-R measurement provides an impedance value (e.g. E/H), usually expressed as apparent resistivity and phase, using short e.g. $5 \mathrm{~m}$ ) electric dipoles. The VLF-R measurement, although appropriate for a one-dimensional (I-D) application, contains only marginal information on the vertical resistivity structure (Fischer et al., 1983) because, in effect, only a single frequency is available. These factors suggest that the strength of VLF methods lies predominantly in the definition of lateral gradients in the subsurface resistivity structure. The interpretation problem is therefore, at least, two-dimensional.

Although extensive and high-density VLF data sets have been used successfully for mapping purposes (e.g. Ogilvy et al., 1991; Tabbagh et al., 1991), techniques which assess the third (i.e. depth) dimension appear both limited and restrictive. Here, as advocated by Fischer et al. (1983), a 2-D modelling (in this case inversion) approach to VLF interpretation is demonstrated using synthetic and field examples. The main aim is to demonstrate an automatic method for the construction of a resistivity cross-section using VLF-R data.

The general problem of EM induction, either by highly-polarised (VLF) or natural-source plane-wave fields requires vector measurements to fully specify the problem. The VLF technique has a directional limitation in that the horizontal magnetic field is conventionally measured in a direction perpendicular to the line of the transmitter and the associated E-field is measured along the line of the transmitter (essentially to ensure maximum signal strength). These limitations and the difficulties of interpretation that ensue have been extensively discussed by Tabbagh et al. (1991).

The close association between the VLF technique and the wide-band, natural-source magnetotelluric (MT) and AMT technique has been noted on many occasions. From the perspective of the MT technique the VLF method presents us with a directional set of VLF-R and/or VLF-Z measurements at a single frequency. Profile data are assumed. The objective is then to determine a resistivity crosssection that fits the measured data. When developing an inverse modelling approach, the recurring pitfalls of inaccurate, insufficient and inconsistent data (Jackson, 1972) must be appreciated. In a 2-D inverse problem which attempts to construct a valid depth cross-section, the use of single frequency 
data guarantees some level of insufficiency (the actual level will be case-specific). Care must also be exercised since the VLF data are directional and the data may be inconsistent with the modes presented by a 2-D problem as outlined below.

As discussed by Fischer et al. (1983), in order to ensure consistency with a 2-D approach, the directional VLF data must conform to one of the two principal modes of 2-D induction. The assumption of infinite strike (which defines the 2-D case) provides two decoupled modes involving separate combinations of the field components. The TE-mode (or E-polarisation, electric field parallel to strike) involves surface fields of $E x, E y$ and $H z$. The TM-mode (or H-polarisation, magnetic field parallel to strike) involves the surface fields $H x$, Ey and Ez. Due to the directional nature of VLF measurements, we require therefore that the measurements be made in, at least, one of the two principal directions. Where the geological strike is not known, the sensible survey option of taking measurements from several azimuthally-distinct transmitters is suggested.

The present study comprises an initial discussion on the developments which have taken place in MT data modelling using regularised inversion techniques. Such procedures provide (in fact impose) smooth models of the subsurface resistivity distribution. These smooth models are intended to provide lower bounds on the amount of structure required to fit the data and, as such, tend to reduce the problem of equivalence that is a component part of employing diffusive EM wavefields. Smooth, minimum-structure resistivity cross-sections are not yet a familiar concept in applied geophysics. In order to provide insight into their practical application to VLF data, both synthetic and field data examples are considered.

\section{Regularised inversion}

The starting point in the modelling of VLF data are the developments in non-linear inversion which have arisen in the context of the multi-frequency MT technique. The new approaches, developed first for the 1-D MT problem and more recently for 2-D geometries, involve regularising an otherwise "ill-posed" problem by introducing a smooth or minimum-structure constraint. In these methods the subsurface resistivity structure is constrained to vary smoothly and we seek the resistivity distribution with the minimum amount of structure (roughness) that fits the observations. The approach is effective because measured data are invariably limited in number and precision (insufficient and inaccurate) and because the EM wavefield is diffusive. These two factors ensure that pointwise discontinuities (e.g. layers/polygons) in the subsurface cannot be resolved without a degree of equivalence. In 2-D modelling and inversion, the problem of equivalence becomes particularly acute because of the larger number of degrees of freedom within the model space. The essential point is that the minimum-structure inversion concept acknowledges this fact and allows the automatic construction of credible (non-extreme) resistivity models.

In the 1-D case, formulations of the inverse MT problem when the vertical resistivity profile is rigorously smooth (e.g. in terms of its first and second derivatives) have been considered by Constable et al. (1987) and by Smith and Booker (1988). For 2-D MT inversion, deGroot-Hedlin and Constable (1990) implemented a minimum-structure inversion which is referred to as OCCAM and is based on the finite-element forward solution of Wannamaker et al. (1987). A more rapid 2-D MT inversion called the Rapid Relaxation Inverse has also been developed by Smith and Booker (1991). Here we use the 2-D OCCAM procedure, described in detail by deGroot-Hedlin and Constable (1990), which has proved to be a very stable algorithm.

It is important that the measured data should possess associated error bounds. The underlying concept is that we should never attempt to obtain an exact fit between measured and modelled 
data. The error bound must comprise the variance associated with physical measurement but it can also encompass the degree to which a particular level of modelling (e.g. I-D, 2-D or 3-D) is thought to be appropriate. Given a set of $N$ observations $\left(o_{i}, i=1, N\right)$ with standard errors $\left(\sigma_{i}\right)$, the concept is to only fit the observations to within a prescribed level of misfit. When the data and errors conform to Gaussian behaviour the chi-square $\left(\mathrm{X}^{2}\right)$ statistic is a natural measure of misfit:

$\chi^{2}=\sum\left|o_{\mathrm{i}}-\mathrm{m}_{\mathrm{i}}\right|^{2} / \sigma_{i}^{2}$

where $m_{\mathrm{i}}$ refers to the $\mathrm{I}^{\prime}$ th model response. For a model fit to be satisfactory we might typically take $\mathrm{X}^{2}<\mathrm{N}+2(2 \mathrm{~N})^{1 / 2}$ (Parker and Whaler, 1981). This would correspond to accepting models whose misfit is less than two standard errors of $X^{2}$ above its expected value of $N$. Here we use an rms measure of misfit defined as $\mathrm{X}^{2} / \mathrm{N}$ with an expectation value of unity.

Smooth, minimum-structure resistivity cross-sections are not yet a familiar concept in applied geophysics. In order to provide some insight into their practical application to VLF data both synthetic and field data are considered. As noted previously, single-frequency profile data present a special case of data insufficiency and it is important to first diagnose the resolution attributes of this special case using synthetic examples.

For consistency, both synthetic examples and field data have been treated using an equivalent formulation of the problem. The field data consist of only 11 observations (along 3 parallel profiles) at $5 \mathrm{~m}$ separations across a $50 \mathrm{~m}$ baseline. The synthetic data, obtained by the forward-modelling scheme of Wannamaker et al. (1987), have been generated at an equivalent set of 11 locations. In all cases, the parameterisation of the inversion model space is the same. The central portion of the model $(50 \mathrm{~m} \times 50 \mathrm{~m})$ consists of a regularisation grid containing individual blocks of a width and thickness of $2.5 \mathrm{~m}$. This parameterisation obviously sets a limit in terms of the minimum-scale of any resolvable features. Both synthetic and field inversion results are presented in true scale across the $50 \mathrm{~m} \times 50 \mathrm{~m}$ central section. Although the inversion grid comprises $2.5 \mathrm{~m} \times 2.5 \mathrm{~m}$ rectangular blocks, results are block contoured at an interval of $1 \mathrm{~m}$. In each case a uniform halfspace is used to initiate the inversion, i.e. we are considering the case of automatic inversion. Constraints which may provide increased resolution are available within the inversion algorithms but are not discussed here.

\section{Synthetic example 1}

This example is primarily intended to demonstrate the nature of minimum-structure cross-sections using one mode and one or two VLF frequencies. It is assumed that two VLF frequencies can be used to provide only a limited frequency separation. Depending on the transmission azimuths, the data might provide results in two separate modes (i.e. both TE and TM and transmission paths orthogonal) or in the same single mode (transmission paths parallel). We here consider the case of observations made at two frequencies ( 16 and $25 \mathrm{Khz}$ ) which provide the same (TE) mode of induction.

The model (see Fig. 2) is a simple conductive zone buried in a half-space. The conductive zone has a resistivity of $5 \mathrm{ohm} . \mathrm{m}$ and is immersed in $100 \mathrm{ohm} . \mathrm{m}$ material. The upper surface of the anomaly is at $10 \mathrm{~m}$ and it extends to $20 \mathrm{~m}$ (i.e. a thickness of $10 \mathrm{~m}$ ). The anomaly is, in effect, a laterally elongate zone (extending to infinity) and we examine the resolution of measurements made over the rectangular terminating edge of the concealed zone. The anomaly has a resistivity contrast of 20 and skindepths in the zone range from $8.89 \mathrm{~m}$ at $16 \mathrm{kHz}$ to 7.11 at $25 \mathrm{kHz}$. The skin-depths at these 
frequencies are comparable to the thickness of the anomaly and a strong inductive response will be

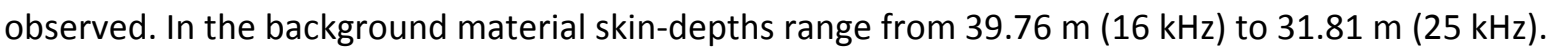
Clearly the electrical scale-lengths are very similar for the two frequencies.

We consider 11 observations made at $5 \mathrm{~m}$ separations, over a total baseline of $50 \mathrm{~m}$. The observational anomaly for the TE-mode at 16 and $25 \mathrm{kHz}$ is shown for apparent resistivity and phase in Fig. 1 (symbols). The edge of the anomaly is situated at - $150 \mathrm{~m}$ in model coordinates (Fig. 2). Examination of Fig. 1 demonstrates that a $50 \mathrm{~m}$ baseline is insufficient to fully define the spatial wavelength of the anomaly. With increasing distance from the edge of the anomaly the response must revert to the corresponding half-space response (an apparent resistivity of $100 \mathrm{ohm} . \mathrm{m}$ and a phase of $45^{\circ}$ ). For this model example the 11 synthetic observations, although accurate, are insufficient in terms of spatial scale.

A series of investigations into the resolution of the data were made using the 2-D OCCAM algorithm. Only the resolution of the TE-mode is reported here. In each case a "blind" starting model consisting of a half-space of $30 \mathrm{ohm} . \mathrm{m}$ was used. Data errors (in the logarithm of apparent resistivity and in linear phase) were set at $1 \%$.The model cross-section obtained at an rms misfit of unity, using the TE-mode at $16 \mathrm{kHz}$ only, is shown in Fig. 2a. The cross-section is in true scale and the logarithm of resistivity is contoured. The target conductive zone is outlined by the heavy dashed line. A blank (white) zone is used here to denote resistivity overshoot, i.e. where model resistivities are less than the target resistivity of $5 \mathrm{ohm}$. $\mathrm{m}$. The lightest gray-scale denotes model resistivities greater than the true background of $100 \mathrm{ohm} . \mathrm{m}$.

The model returned by the inversion is necessarily smooth and consists of a series of gradients with associated spatial wavelengths. In very broad terms the minimum-structure solution can be seen to be "generally" consistent with the starting model when given (1) accurate data (i.e. synthetic and nonoise data) and (2) insufficient and inadequate data (i.e. only one mode, one frequency and a limited spatial wavelength). The resolution obtained in this example can be summarised as follows. The first reflecting horizon (the upper surface of the conductive zone) is the best resolved feature. Since only one frequency is used to constrain the solution, subsequent vertical resolution (e.g. the lower surface of the conductive zone) is more limited. The most conductive feature of the solution is likely to be displaced downwards from the "true" centre and inwards from the "true" edge. The "edge effect" consists of a set of gradients that are "more realistic" in the upper portion of the section. The resolution through the lower part of the section is very limited.

It should be noted that a highly accurate solution can be obtained by the OCCAM inversion, particularly using synthetic data. This is achieved by providing multi-frequency profile data that "best-suit" the electrical scale of the problem. For our present purposes we wish to consider typical resolution attributes of the limited type of data that would be supplied by routine VLF data sets.

A model cross-section obtained using the TE-mode data and the two VLF frequencies of 16 and 25 $\mathrm{kHz}$ is shown in Fig. $2 \mathrm{~b}$. The rms misfit of the solution is again unity. The introduction of the higher frequency (in the same mode) in this example has increased the resolution of the target, particularly through the upper part of the section but also generally throughout the section. In this example, near-surface resistivities of the correct half-space value $(100 \mathrm{ohm} . \mathrm{m})$ are returned by the inversion and the most conductive feature is centred on the target anomaly. The lowest individual $(2.5 \times 2.5$ $\mathrm{m}$ ) block resistivity in the conductive zone is $3.5 \mathrm{ohm} \mathrm{m}$ which compares favourably with the actual value of $5 \mathrm{ohm}$. $\mathrm{m}$. The response of the model for both frequencies and an rms misfit of unity, is compared with the observed synthetic data set in Fig. 1. It should be noted that the misfit is an "overall" figure and is, inevitably, distributed over the 44 observations. It can be seen that there is 
scope for further matching between the observed and modelled data, particularly in the phase response at $25 \mathrm{kHz}$.

For this example it has proven possible to "overfit" the synthetic data by a factor of two, producing a stable solution at an rms misfit of 0.5 with the same $1 \%$ errors assigned to the data. The resulting model, using the same data as in the previous case is shown in Fig. 2c. The increase in structural resolution over the previous case is only marginal.

\section{Synthetic example 2}

This example is primarily intended to demonstrate the nature of minimum-structure cross-sections using one or two of the different modes that may be observed at a single frequency. The anomaly studied is more complex than that of the previous case in that it consists of an isolated conductive zone dipping at about $45^{\circ}$. The structure, which simulates a dipping fault zone, is a typical application for VLF observations. The model was developed as a control for the interpretation of the field data modelling discussed later. The model (see Fig. 5) is again a simple conductive zone of 5 ohm.m immersed in a material of $100 \mathrm{ohm}$. $\mathrm{m}$. The top of the zone is set at $2 \mathrm{~m}$ and has a width of 5 $\mathrm{m}$. The zone dips at an angle of about $45^{\circ}$ down to a depth of $20 \mathrm{~m}$. The scale of the anomalous structure has dimensions ( 5 and $18 \mathrm{~m}$ ) both less than and greater than one skin-depth of a $16 \mathrm{kHz}$ wavefield within the anomalous zone $(8.89 \mathrm{~m})$.

We again consider 11 observations made at $5 \mathrm{~m}$ separations over a total baseline of $50 \mathrm{~m}$ which is centred above the anomalous region. The observational anomaly for the TE- and TM-modes at 16 $\mathrm{kHz}$ is shown in Fig. 3. The vertical field ( $\mathrm{Z}$ ) response (real and imaginary components) produced in the TE-mode is shown in Fig. 4. It should be noted that in Figs. 3 and 4 the solid lines are simply data traces (they are not inversion results). The asymmetry in all the response data, produced by the dipping anomaly, is very evident.

For this type of anomaly, the spatial wavelengths of the anomalous response decrease as TMmode $<T E$-mode $<Z$ (TE-mode). For a sufficient coverage of the anomalous region we would require the apparent resistivities to return to $100 \mathrm{ohm}$. $\mathrm{m}$, the phases to return to $45^{\circ}$ and the Z-field to return to zero. As can be seen in Figs. 3 and 4 this does not occur using an observational baseline limited to $50 \mathrm{~m}$.

As in the previous example, a series of investigations into the resolution of the data were made using the 2-D OCCAM algorithm. Again, a "blind" starting model consisting of a half-space of $30 \mathrm{ohm}$. $m$ was used throughout. Data errors of $1 \%$ were again assumed. Although "more accurate" solutions have been obtained, the solutions obtained at an rms misfit of unity are presented. It will be noted that the contour level scheme is almost the same as that used in the previous example (model resistivities are the same). Here a lower bound of 0.75 (logarithmic scale) is used since, for this example, modelled resistivities approach, but do not achieve, the target resistivity of $5 \mathrm{ohm} . \mathrm{m}$.

The model cross-section obtained at an rms misfit of unity using the TE-mode data (apparent resistivity and phase) at $16 \mathrm{Khz}$ is shown in Fig. 5a. The cross-section is true scale and the logarithm of resistivity is contoured. The target conductive zone is outlined by the heavy dashed line. With only one frequency constraining the solution, the imaging of a dipping zone will inevitably be difficult. In broad terms, the smooth solution returns an asymmetrical conductive zone. The upper surface of the zone (first reflecting horizon) is well resolved and the upper surface of the dipping zone is imaged by a gradient whose dip is close to that of the target zone. Elsewhere, through the upper part of the section, the trace of the most conductive zone in the model is closer-to-vertical than the 
actual dip. As was the case in the previous model, the resolution through the lower part of the section is very limited.

The equivalent cross-sectional model obtained using the TM-mode data is shown in Fig. 5b. The model obtained has broadly similar characteristics to that obtained from the TE-mode data. In detail, the set of gradients in the vicinity of the low resistivity zone appear to "contain" the true dip in a more satisfactory manner. The modelling investigation of the TE-mode data can be extended by assuming that vertical field data are also available. This increases the number of observational constraints by a factor of 2 (from 22 to 44 , for the 11 site profile). The cross-sectional model obtained using the TE-mode $R$ and $Z$ data at $16 \mathrm{kHz}$ is shown in Fig. 5c. The increase in true structural resolution over that obtained using only the TE-mode $\mathrm{R}$ data is only marginal.

As in the previous example, we might also assume that two VLF frequencies can be recorded. In this final synthetic example we consider the case in which observations of two frequencies (15 and 30 $\mathrm{kHz}$ ) have been obtained in the same TM-mode (i.e. the VLF transmissions had very similar azimuths). As in the previous example, it should be noted that two frequencies provide similar skindepths in the two model regions. The resulting model, obtained at a misfit of unity, is shown in Fig. $5 \mathrm{~d}$. The cross-section probably represents the "most-realistic" image obtained, thus far, by the minimum-structure inversion. The image of an isolated dipping conductive zone in an otherwise uniform background is clearly well-represented by the gradients obtained. Once again the upper surface of the conductive zone (first wavefield contact with the anomaly) is the best-imaged feature. The "more-resistive" zone to the right of the true anomaly is thought to be due to Gibb's phenomenon (i.e. trying to fit a smooth model to a feature exhibiting sharp discontinuities can result in model overshoot).

In summary, for the dipping model studied, complete resolution of the "true" structure is difficult when only one VLF frequency is involved. For the model studied both TE- and TM-mode data provide similar resolution attributes. As in the previous example, the first reflecting horizon is the best resolved feature. The upper surface of the dipping zone is then imaged by a gradient whose dip is close to that of the target zone. A series of "false", near-vertical gradients project downwards from the upper surface. In all cases the minimum resolved resistivities occur within the anomalous zone, close to the upper surface. The values of the lowest resistivities appear to be marginally higher than the true resistivities.

\section{Application to field data}

The data studied here were collected as part of a collaborative study into geophysical methods for the recognition of geological weakness zones and other surface discontinuities caused by underground mining in Carboniferous terrain (Greenwood and Peart, 1992). In one of the study areas (Saar 2.1, Test Site 3) three profiles of VLF-R and -Z measurements were made. The three profiles are shown in Fig. 6 (plan-view) and were made along azimuths of N59E. The three profiles are $50 \mathrm{~m}$ in length, are separated by $10 \mathrm{~m}$, and each comprise 11 measurements made at $5 \mathrm{~m}$ intervals. The profiles are referred to as 00S, $10 \mathrm{~S}$ and 20S. The lateral, along-profile, coordinates range from - 175 to $-125 \mathrm{~m}$. Also shown in Fig. 6 is a possible fault trace based on a $10 \mathrm{~cm}$ topographic feature. 
The measurements were made with the Scintrex IGS-2 equipment, using $5 \mathrm{~m}$ E-field dipoles and the Rugby (UK) VLF transmitter (GBR, $16 \mathrm{kHz}$ ). The transmission azimuth, along which E-field dipoles were aligned was $320^{\circ}$. The equipment has a reading resolution of $1 \%$ in the case of the VLF-Z components and 1 ohm.m and $1^{\circ}$ in the case of the VLF-R measurements. The map of Fig. 6 also displays the in-phase $Z$ results that were obtained. Both in-phase and in-quadrature (not shown) $Z$ fields are small and consistently less than $7 \%$ of the associated horizontal component. In addition, it can be noted from Fig. 6 , that there is no convincing (i.e. strong) sign reversal in the in-phase component. Such a characteristic is the normal signature when traversing a lateral discontinuity (e.g. Fig. 4).

The VLF-R data obtained along the 3 profiles are shown in Fig. 7a-c. The observational results are indicated by symbols and the full and broken lines are model results which are discussed later. As explained previously it is necessary to assign errors to the data observed. The data shown in Fig. 7a-c have had "nominal" 2.5\% error bounds applied (in the logarithm of apparent resistivity and in linear phase). Despite the separation of only $10 \mathrm{~m}$ between successive profiles there are very clear differences in the behaviour of the response characteristics across the three sets of observations. A clear minimum in apparent resistivity is observed along profiles $00 \mathrm{~S}$ and $10 \mathrm{~S}$ but along $20 \mathrm{~S}$ the form of response is a step. The phase response along profiles OOS and $10 \mathrm{~S}$ shows a clear similarity (in both magnitude and form) but the correspondence is not mirrored in the behaviour of the two sets of apparent resistivities. These differences would, in practice, make a combined interpretation across the three profiles difficult.

In contrast to 1-D modelling and inversion of VLF data (Mathieson and Crossley, 1982), 2-D modelling and inversion requires the appropriate mode of the data to be established. The choice of whether the observed data are responding in either the TE-mode or TM-mode will inevitably be subject to error unless an unambiguous anomaly strike direction is known and measurements are then made using VLF transmitters in principal strike coordinates (i.e. perpendicular and/or parallel to strike). Even in the case, as here, where a small topographic feature at the surface is thought to relate (in some unknown way) to the fault at depth, it should be noted that the VLF wavefield at 16 $\mathrm{kHz}$ may effectively respond to resistivity gradients to a considerable depth (e.g. $>20 \mathrm{~m}$ ).

A great deal of insight into the procedure for establishing the correct mode of the data can be obtained using the response characteristics of synthetic models such as those presented in the previous sections. Referring to Fig. 3 (synthetic example 2), both the TE- and TM-mode responses in apparent resistivity display minima in the vicinity of the resistivity contrast. In such a case (i.e. a conductive anomaly) the TM-mode phase will display a characteristic increase (to a maximum) while the TE-mode phase displays a corresponding decrease to provide a minimum. Thus the phase information can be diagnostic of the induction mode. In this case we can compare the synthetic TEand TM-mode phase response (Fig. 3) with that of the observed data (Fig. 7a-c). In Fig. 7a and b it can be seen that phase maxima are observed on profiles $00 \mathrm{~S}$ and $10 \mathrm{~S}$ in association with minima in the amplitude responses and as such should be associated with a TM-mode response.

The phase behaviour along profile 20S, although less convincing, is still compatible with TM-mode behaviour. The Z-field data, when available, can provide an additional constraint with regard to mode identification. A Z-field can only be generated in the case of a TE-mode response. It is entirely absent in the case of pure TM-mode induction (under the 2-D assumption). The Z-field response observed in the TE-mode across the second synthetic model was shown in Fig. 4. Here it can be noted that the in-phase response changes sign at the location where the minima in apparent resistivities are observed. Maxima and minima in the Z-field are then observed to either side of the 
phase change. The essential point is that in the case of a TE-mode response which gives rise to a significant lateral VLF-R response, an associated VLF-Z response must always exist.

In the case of the present survey data, the measured VLF-Z response is limited to values $<0.07$ (Fig. 6). This fact, together with the characteristic VLF-R phase behaviour noted above confirms that the measured data conform, quite closely, to a TM-mode response. Using the data shown by symbols in Fig. 7a-c, a series of investigations into the resistivity cross-sections were made using the 2-D OCCAM algorithm, under the assumption that the data represent a TM-mode response. As in the previous synthetic examples, a "blind" starting model of $30 \mathrm{ohm} . \mathrm{m}$ was used in each case. Using the nominal $2.5 \%$ error assignments, it was found that the data from all three profiles could be inverted in one or two iterations to provide an rms misfit of unity. The responses of the models obtained by this procedure are shown by the broken lines in Fig. 7a-c.

The three resistivity cross-sections obtained are shown in Fig. 8a (profile 00S), b (profile 10S) and c (profile 20S). All three cross-sections display smooth resistivity transitions between "conductive" regions of order $25 \mathrm{ohm} . \mathrm{m}$ through to more resistive regions with resistivities $>100 \mathrm{ohm}$.m. The cross-sections obtained for profiles $10 \mathrm{~S}$ and $20 \mathrm{~S}$ (Fig. $8 \mathrm{~b}$ and c) show a similar form, i.e. a nearsurface resistivity low centred on - 155 in Fig. 8b, transfers to a more westerly (i.e. to the left) position in Fig. 8c. In the case of profile 00S (Fig. 8a) the near-surface conductive feature is centred on - 140 and the cross-section involves less steeply-dipping gradients. Resolution in minimum structure inversion is a function of misfit. An examination of the model fits obtained at unity misfit (Fig. 7a, b) indicates that, particularly in the case of the phase data, the lower-order data wavelengths have only been partially reproduced. We could, in fact, regard the cross-sections associated with these fits as "conservative" estimates of the most likely resistivity distribution. The OCCAM algorithm is, in general, capable of providing a cross-section that fits most of the fine-detail of valid observational data. With decreasing misfit, however, we must eventually start to model noise.

The observational data can be viewed as containing a superposition of wavenumber components. The fitting of the low wavenumber components to an rms misfit of unity provided the cross-sections which can be seen to contain only long wavelength gradients. High-wavenumber features in the data (e.g. gradients at the measurement separation scale) can only be modelled by short-wavelength features in the cross-section. In cases where such gradients are "noise", artificial model features, particularly in the shallow sub-surface, will inevitably be produced. These points are welldemonstrated by the three data sets considered here. Fig. 9a-c shows the three resistivity cross sections obtained by reducing the misfit level to 0.25 (with assumed data errors remaining at $2.5 \%$ ). The associated response data are compared with the observed data by the solid lines in Fig. 7a-c. Here it can be seen that a degree of "overfit" has taken place in providing models which fit the data "exactly". The cross-sections now contain some high-wavenumber features which, particularly within the upper $10 \mathrm{~m}$, are most likely to be "noise". Some of the lower wavenumber features are, however, stable developments of features observed previously at the lower level of misfit.

For the data sets considered here, the most realistic cross-sections lie within the "limits" provided by the two sets of results (i.e. at misfits of 1 and 0.25 ). Both sets can be used for interpretation purposes with the 0.25 level results requiring a much higher degree of caution. It can be noted from Fig. 9a and $b$ that the characteristic dipping conductive feature is compatible with that imaged in the second synthetic model analysis. The previous (forward) model analyses therefore represent an important aspect of interpretation control. The results from the southernmost profile $20 \mathrm{~S}$ (Fig. 9c) do not preclude the same type of feature. In the case of 20S, the same (assumed) feature appears to lie too close to the edge of the observational profile to allow the "same degree" of resolution. 
Using the synthetic model results as a control, the interpretation of features observed in the resistivity cross-sections can be undertaken. A subsurface conductive zone, assumed to be faultrelated, occurs on all three cross-sections. The lowest resistivity within the zone (averaged over $2.5 \mathrm{x}$ $2.5 \mathrm{~m}$ ) is of the order of 15- $20 \mathrm{ohm}$. $\mathrm{m}$. Away from the immediate vicinity of this zone at- and nearsurface resistivities return to values in excess of $100 \mathrm{ohm}-\mathrm{m}$. The upper surface of the zone lies some 3-5 m beneath the surface. On profiles OOS and $10 \mathrm{~S}$ the position of the upper surface of the conductive zone lies directly beneath a previously identified topographic feature. The resolution of (possibly) the same zone on profile $20 \mathrm{~S}$ is made difficult due to the lateral limits of the observations, On profiles $00 \mathrm{~S}$ and $10 \mathrm{~S}$ the conductive feature has a strong component of dip that probably exceeds $45^{\circ}$ in both cases. Using observations at $5 \mathrm{~m}$ separations, our resolution of the true width of the zone is limited. Although the intrinsic resolution through the deeper part of the section is poor, on the basis of the control model results, it is possible to suggest that the conductive feature maintains a significant resistivity contrast only to depths of the order of 20-30 m.

\section{Conclusions}

VLF-EM observations (both VLF-Z and VLF-R) provide profile information which usually require to be modelled by a 2-D cross-section of resistivity structure. Viewed from the perspective of other, multifrequency EM techniques the VLF method is a high-density but essentially single-frequency method. These characteristics present data modelling techniques, particularly that of data inversion, with a special case of observational insufficiency. The 2-D data modelling described here imposes additional demands on the procedures of directional VLF data acquisition (to avoid inconsistency with the 2-D approach). Where geological strike is not known, measurements need to be made using two or more azimuthally-distinct transmitters.

The application of a 2-D regularised inversion scheme, to both synthetic and field data, has demonstrated that the inherent data insufficiency can be compensated to some degree, by imposing the constraint of minimum-structure. Regularised inversion schemes are capable of the automatic construction of credible resistivity models. The results demonstrate that the resistivity gradients contained in such models must still be interpreted with care. A single frequency EM technique can only provide a limited vertical resolution. The synthetic studies indicate that the zone of first wavefield contact with an anomalous region produces the highest level of structural resolution. Beneath this region, with increasing depth, resolution must inevitably decrease. Forward resolution studies of type-examples, to clarify the interpretation of inverted field data, are recommended.

\section{Acknowledgements}

The research was funded in part by the Commission of the European Communities (EC Contract No. 7220- AF/136). My thanks go to Catherine deGroot-Hedlinwhose OCCAM2 algorithm provided the motivation for this study. The report is published with the approval of the Director, British Geological Survey (NERC).

\section{References}

Constable, S.C., Parker, R.L. and Constable, C.G., 1987. Occam'sinversion: a practical algorithm for generating smooth models from EM sounding data. Geophysics, 92: 289-300.

deGroot-Hedlin, C.M. and Constable, S.C., 1990. Occam's inversion to generate smooth, twodimensional models from magnetotelluric data. Geophysics, 55: 1613-1624.

Fischer, G., Le Quang, B.V. and Muller, I., 1983. VLF ground surveys, a powerful tool for the study of shallow two-dimensional structures. Geophys. Prospect., 31: 977-991. 
Greenwood, P.G. and Peart, R.G., 1992. Methods for Recognition of Geological Weakness Zones and Other Surface Discontinuities Caused by Underground Mining in Carboniferous Terrain. Br. Geol. Surv. Techn. Rep., WN/93/02C.

Jackson, D.D., 1972. Interpretation of inaccurate, insufficient and inconsistent data. Geophys. J.R. Astron. Soc., 28:97-110.

Mathieson, C. and Crossley, D.J., 1982. Interpretation of single frequency VLF data. In: L.S. Collett and O.G. Jenson (Editors), Geophysical Applications of Surface Wave Impedance Measurements. Geol. Surv. Can., Pap. 81-15, pp. 49-65.

McNeill, J.D., 1990. Use of electromagnetic methods for groundwater studies. In: S.H. Ward (Editor), Geotechnical and Environmental Geophysics, 1: Review and Tutorial. Soc. Explor.Geophys., Tulsa, OK, pp. 191-218.

Ogilvy, R.D., Cuadra, A., Jackson, P.D. and Monte, J.L., 1991.Detection of an air-filled drainage gallery by the VLF resistivity method. Geophys. Prospect., 39: 845-859.

Parker, R.L. and Whaler, K,A., 1981. Numerical methods for establishing solutions to the inverse problem of electromagnetic induction. J. Geophys. Res., 86: 9574-9584.

Smith. J.T. and Booker, J.R., 1988. Magnetotelluric inversion for minimum structure. Geophysics, 53: 1565-1576.

Smith, J.T. and Booker, J.R., 1991. Rapid inversion of two- and three-dimensional magnetotelluric data. J. Geophys. Res., 96: 3905-3922.

Strangway, D.W., Swift, Jr, C.M. and Holmer, R.C., 1973. The application of audio-frequency magnetotellurics (AMT) to mineral exploration. Geophysics, 38:1159-1175.

Tabbagh, A., Benderitter, Y., Andrieux, P., Decriaud, J.P. and Guerin, R., 1991. VLF resistivity mapping and verticalization of the electric field. Geophys. Prospect., 39:1083-1097.

Wannamaker, P.E., Stodt, J.A. and Rijo, L., 1987. A stable finite element solution for two-dimensional magnetotelluric modelling. Geophys. J.R. Astron. Soc., 88: 277-296. 


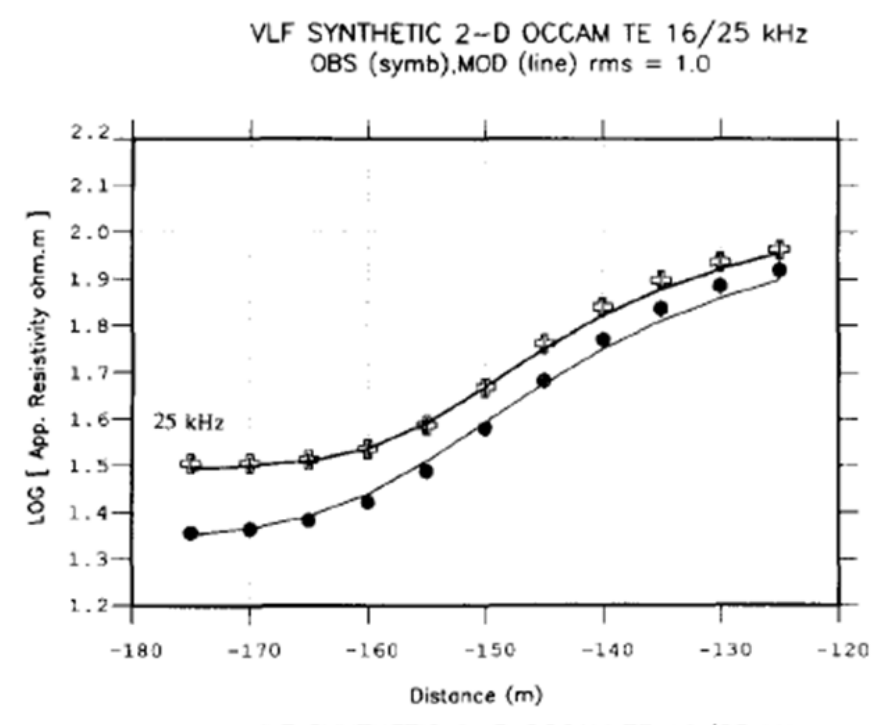

VLF SYNTHETIC 2-D OCCAM TE $16 / 25 \mathrm{kHz}$ OBS (symb), MOD (line) $\mathrm{rms}=0.5$

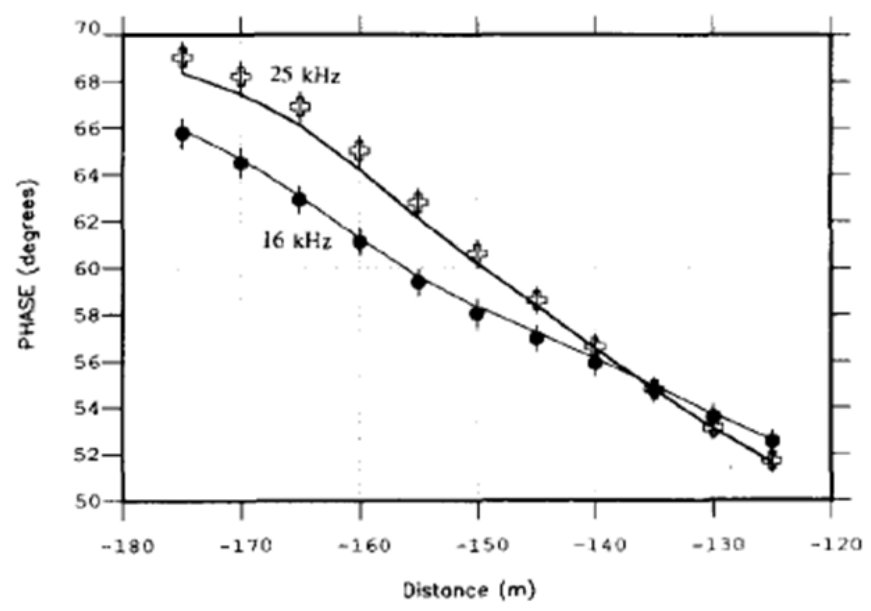

Fig. 1. VLF-R response of synthetic model 1 at $16 \mathrm{kHz}$ (solid circle symbols) and $25 \mathrm{kHz}$ (open cross symbols) at 11 locations (- 175 to $-125 \mathrm{~m}$ ). Error bars denote $1 \%$ error bounds assigned. Solid lines are inversion model results discussed in the text. Apparent resistivity, on logarithmic scale (upper diagram), phase (lower diagram). 

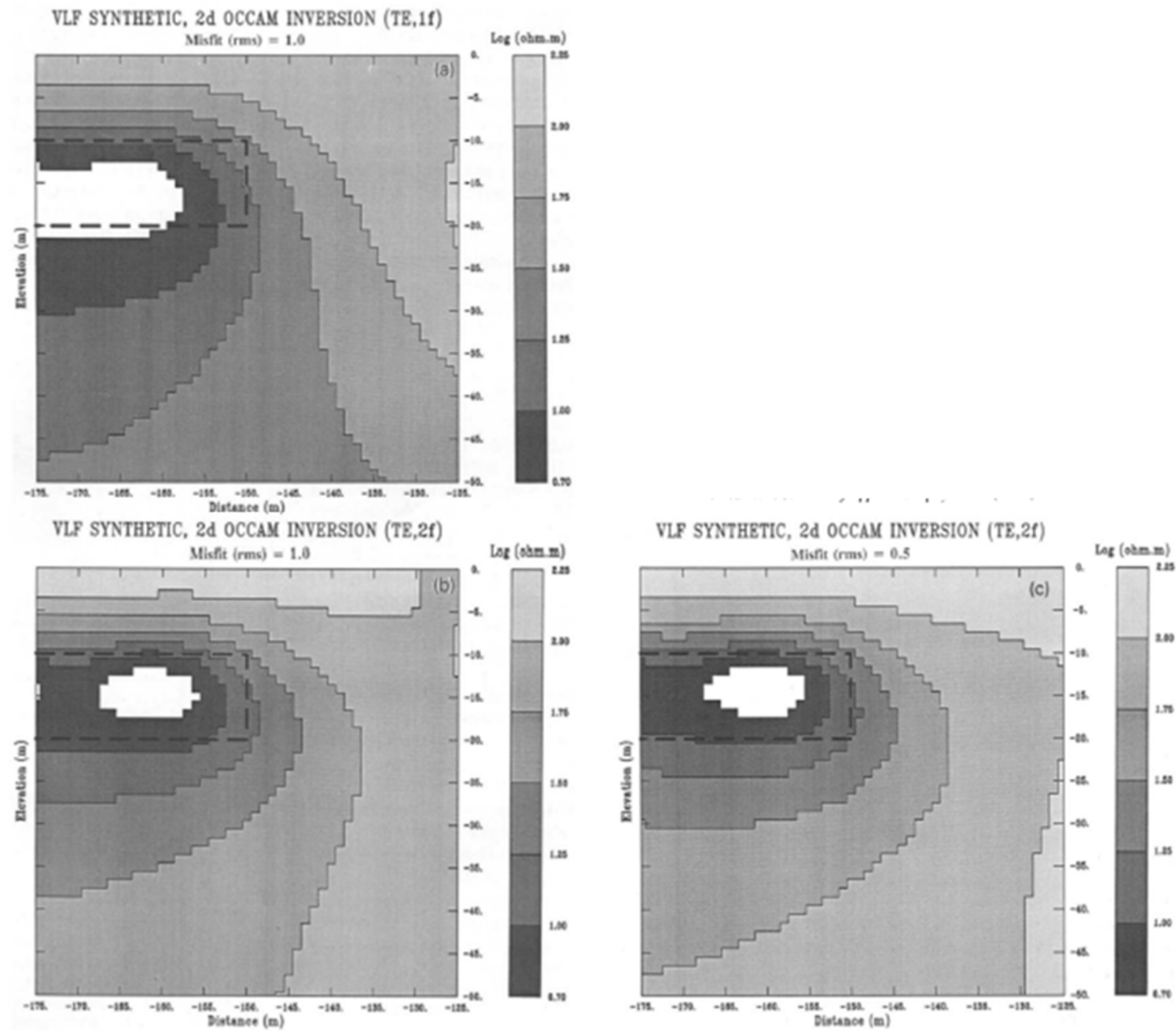

Fig, 2. Smooth resistivity solutions obtained by inverting the TE-mode data of synthetic model 1 . The model is shown outlined by the heavy dashed line. Results contoured are the logarithm of the resistivity using a contour interval of 0.25 (except for lowest bound). The blank (white) zone corresponds to modelled resistivities $<5 \mathrm{ohm}$ - $\mathrm{m}$. True scale cross-section. (a) Model obtained using a single frequency of $16 \mathrm{kHz}$. misfit $(\mathrm{rms})=1.0$. (b) Model obtained using two frequencies of 16 and $25 \mathrm{kHz}$. Misfit $(\mathrm{rms})=1.0$. (c) Model obtained using two frequencies of 16 and $25 \mathrm{kHz}$. Misfit (rms) $=0.5$. 

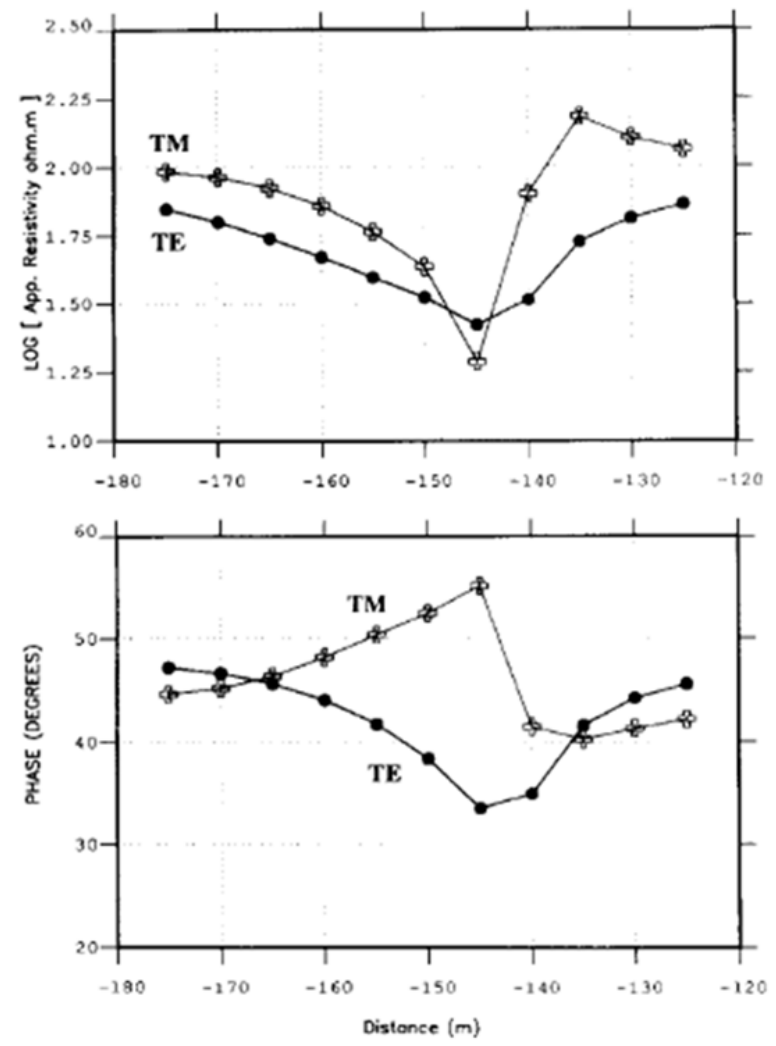

Fig. 3. VLF-R response of synthetic model 2 at $16 \mathrm{kHz}$, TE-mode (joined solid circles), TM-mode (joined open crosses) at 11 locations (- 175 to $-125 \mathrm{~m}$ ). Error bars ( $1 \%$ error bounds) are less than size of symbols. Apparent resistivity, on logarithmic scale (upper diagram), phase (lower diagram). 


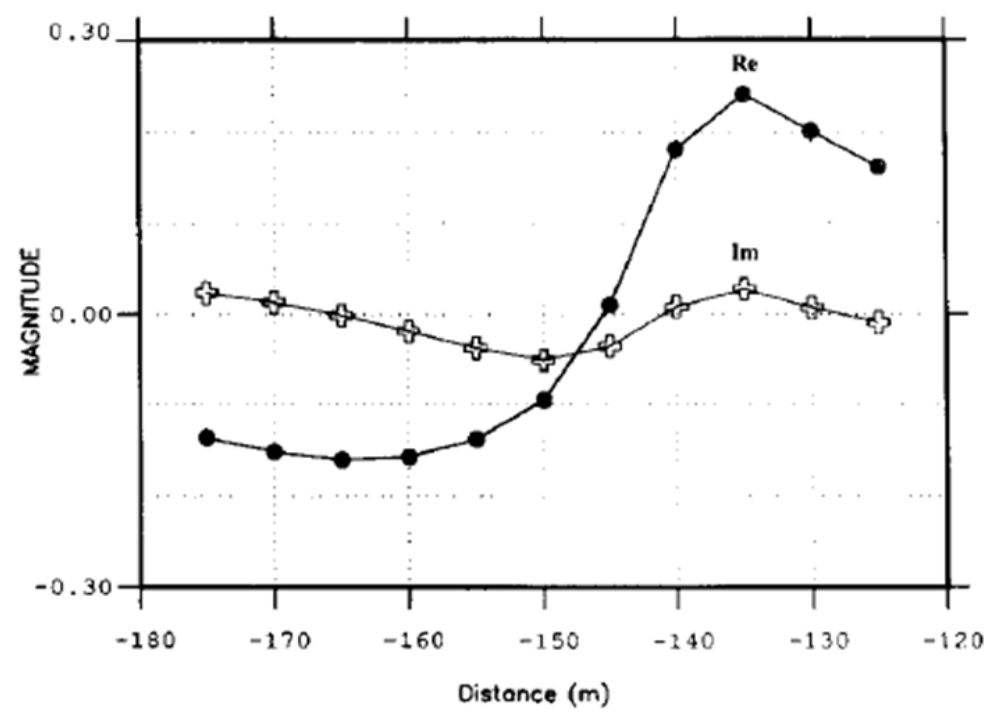

Fig. 4. VLF-Z response of synthetic model 2 at $16 \mathrm{kHz}$, TE-mode. In-phase component (joined solid circles), in-quadrature component (joined open crosses) at 11 locations (- 175 to - $125 \mathrm{~m}$ ). Error bars ( $1 \%$ error bounds) are less than size of symbols. 

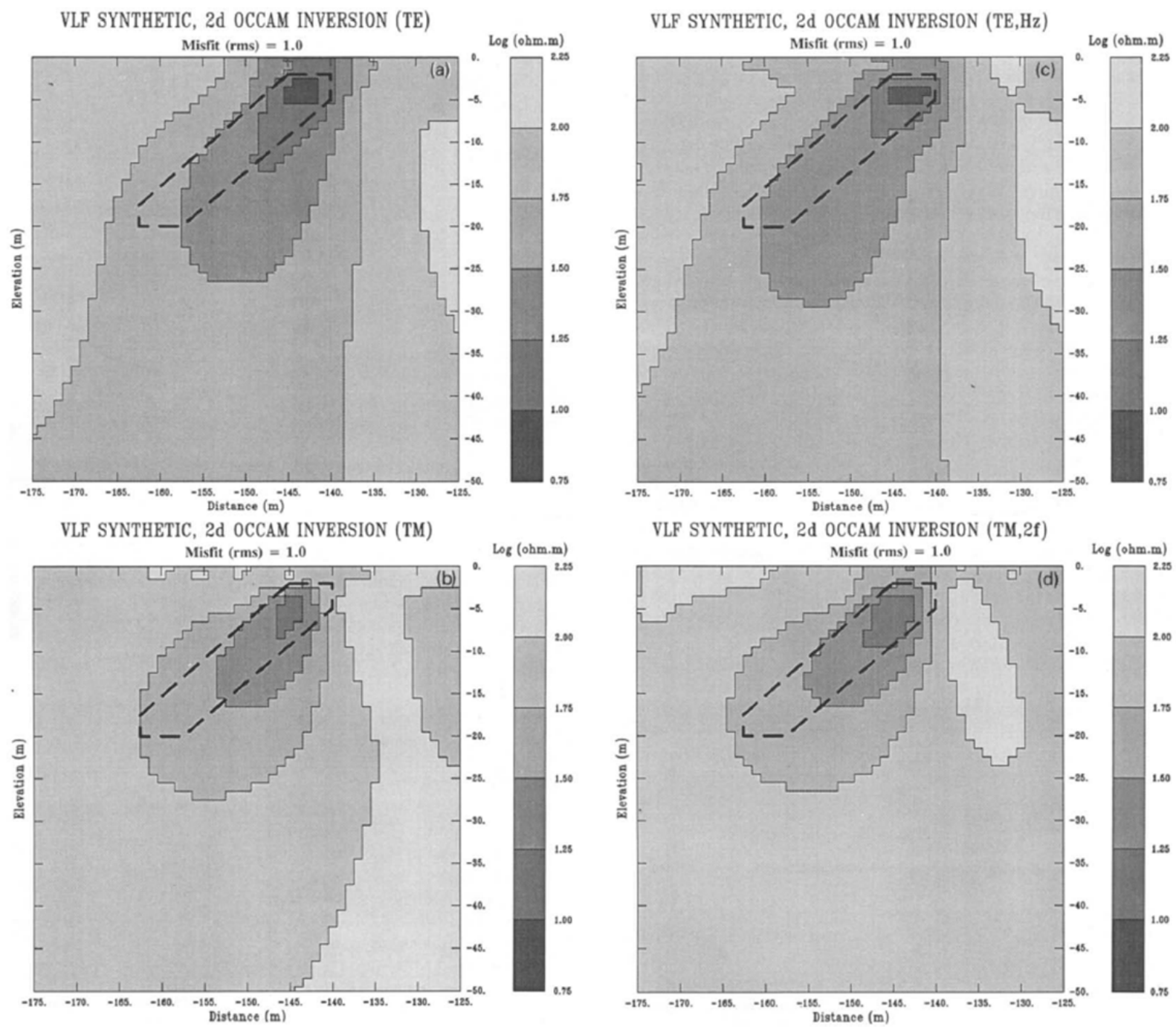

Fig. 5. Smooth resistivity solutions obtained by inverting the test data of synthetic model 2 . The model is shown outlined by the heavy dashed line. Results contoured are the logarithm of the resistivity using a contour interval of $\mathbf{0 . 2 5}$. True scale cross-section. The rms misfit is $\mathbf{1 . 0}$, in each case. (a) TE-mode, VLF-R data. Frequency $=16 \mathrm{kHz}$. (b) TM-mode, VLF-R data. Frequency= $16 \mathrm{kHz}$. (c) TE-mode, VLF-R and VLFZ data. Frequency $=16 \mathrm{kHz}$. (d) TM-mode, VLF-R data. Frequencies = 16 and $25 \mathrm{kHz}$. 


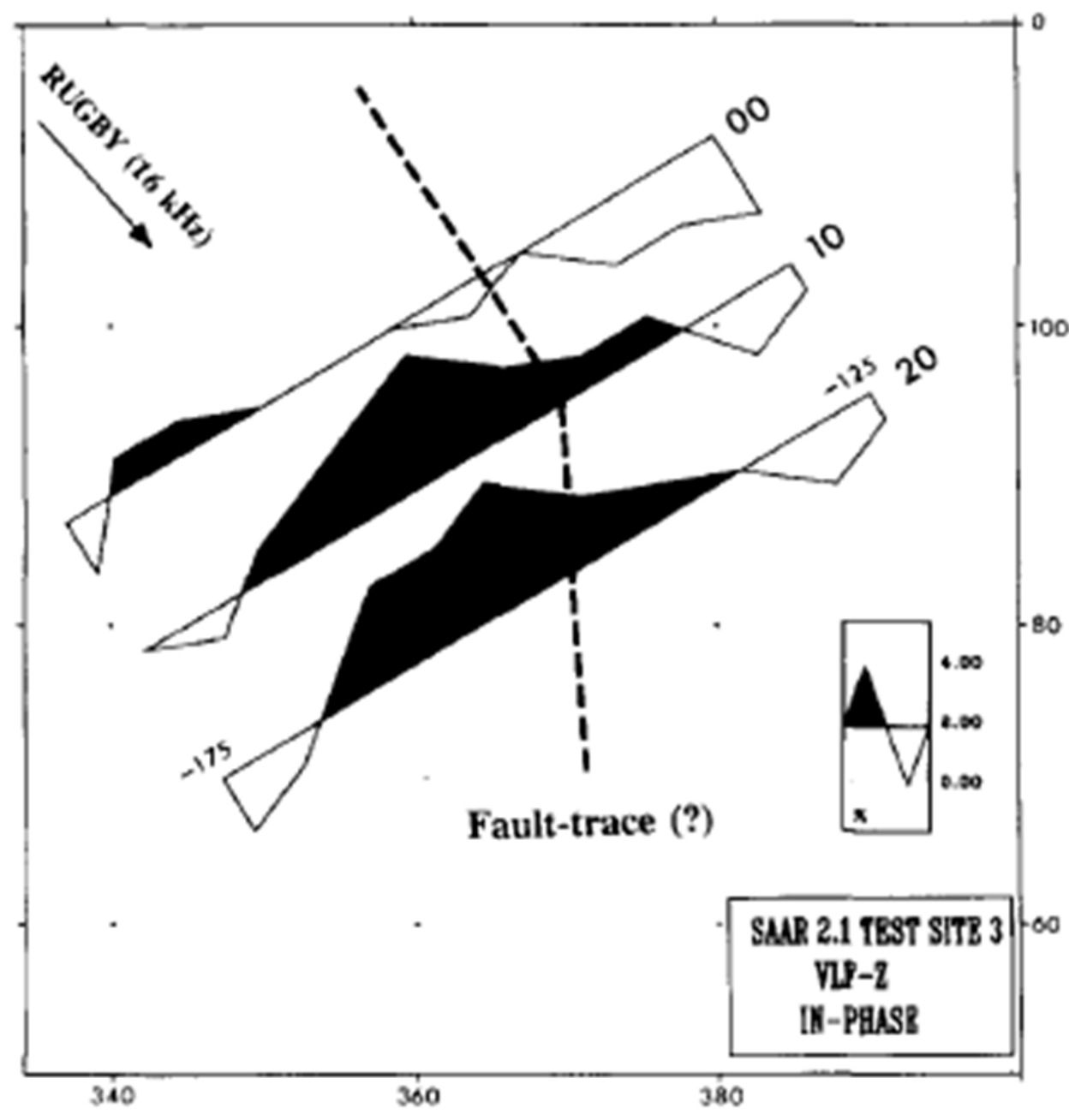

Fig. 6. Plan view of three profiles of VLF measurements. The three profiles are referred to as $00 \mathrm{~S}$, $10 \mathrm{~S}$ and 20S. The fault-trace (?) is a component expressed as a percentage. Grid and profile units are in metres. 

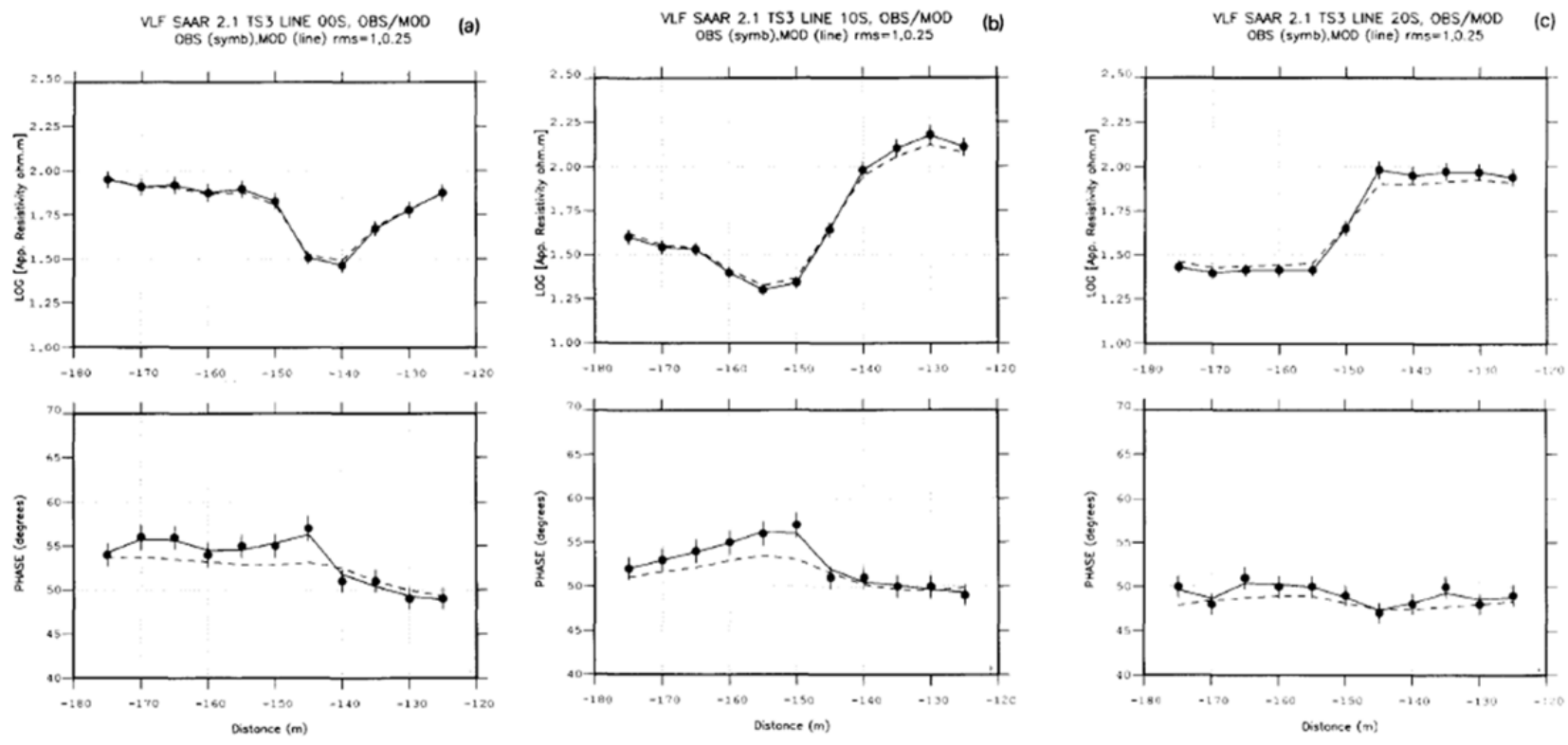

Fig. 7. VLF-R data. The solid symbols are the observed data. Error bars are $2.5 \%$ error bounds. The connecting lines are inversion model results. Dashed line ( $r m s$ misfit of 1.0), full line ( $r m s$ misfit of 0.25). (a) Profile 00S. (b) Profile 10S. (c) Profile $20 \mathrm{~S}$. 

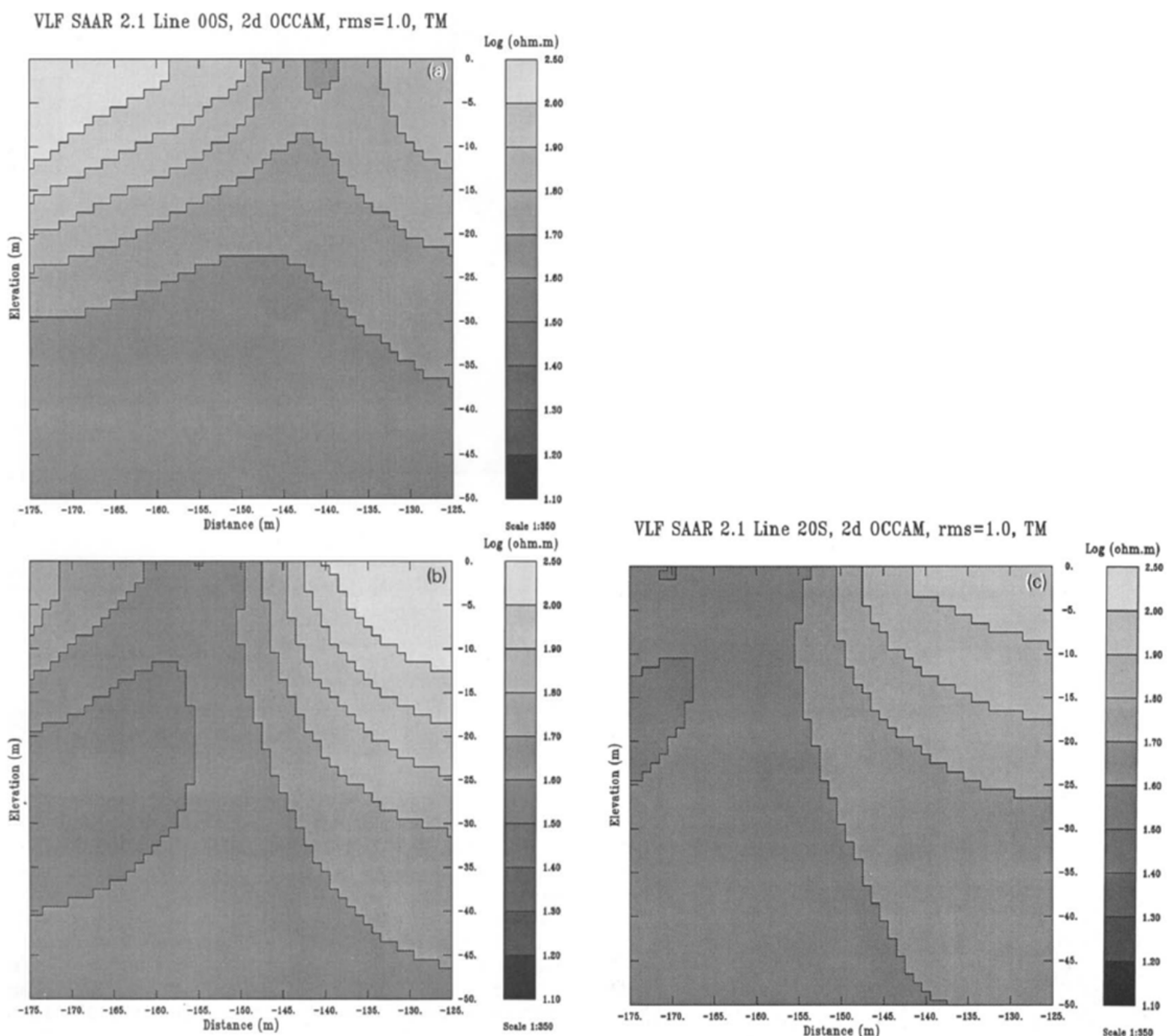

Fig. 8. Smooth resistivity solutions obtained by inverting VLF-R profile data. Results contoured are the logarithm of the resistivity using a contour interval of 0.1 . True scale cross-section. The rms misfit is 1.0, in each case. (a) Profile 00S. (b) Profile 10S. (c) Profile $20 \mathrm{~S}$. 
VLF SAAR 2.1 Line 00S, 2d 0CCAM, rms=.25, TM

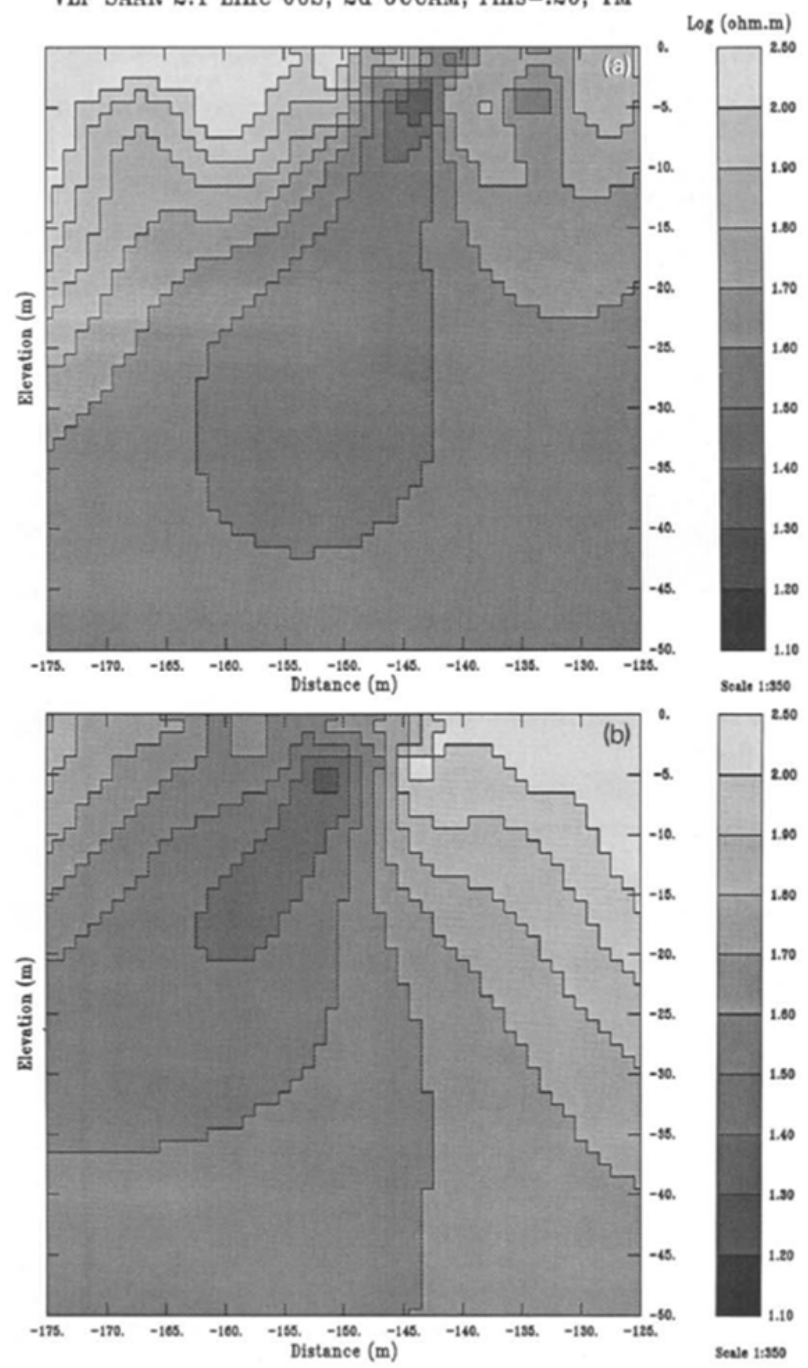

VLF SAAR 2.1 Line 20S, 2d 0CCAM, rms=.25, TM

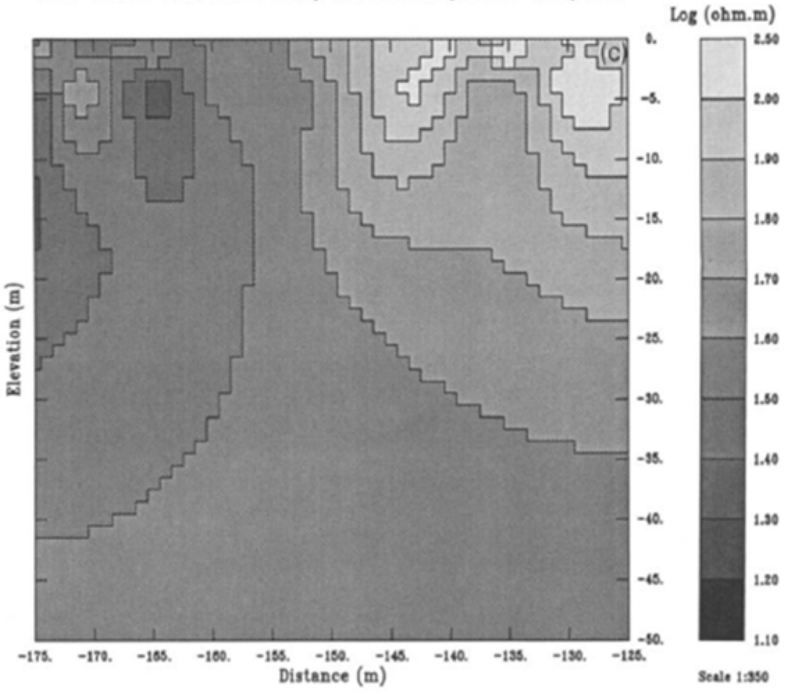

Fig. 9. Smooth resistivity solutions obtained by inverting VLF-R profile data. Results contoured are the logarithm of the resistivity using a contour interval of 0.1 . True scale cross-section. The rms misfit is 0.25 , in each case. (a) Profile 00S. (b) Profile 10S. (c) Profile $20 \mathrm{~S}$. 